\title{
Lettre à la rédaction : Les cheveux de Napoléon, source externe ou ingestion d'arsenic?
}

\section{Napoleon's hair, external arsenic or arsenic ingestion?}

Thomas HINDMARSH, Université d'Ottawa, Philip F. CORSO, Université de Yale

(Reçu le 20 mai 2002 ; accepté le 23 mai 2002)

We do not agree that Kintz et al(1) have confirmed that the arsenic in Napoleon's hair was derived from oral ingestion. They showed that an acetone-water wash removed from human hair the arsenic derived from 4 hours suspension in an $\mathrm{As}_{2} \mathrm{O}_{3}$ solution. Perhaps if they had exposed the hair to arsenic for longer it would not have been removed. Smith and Henry(2) were only able to remove $40 \%$ of arsenic derived from 10 days soaking of human hair in a sodium arsenite solution, by washing in water and sodium hydroxide. Also Young and Rice(3) were unable to distinguish between 'internal' and 'external' arsenic in guinea-pig hair by washing in acid, alkali, ethanol, or ether. Atalla(4) concluded the same using human hair. We have shown(5) that externally applied arsenic can sometimes be found in the core of human hair and it is difficult to imagine how this could be accessible to cleansing solutions.

Kintz et al(1) explain the major differences in arsenic content of the Emperor's hair on the same day, some normal, some lethal, as a consequence of the large variations in inter-hair and intra-hair arsenic. This is a cogent point which emphasizes the unreliability of single-hair arsenic measurements(6). It is essential that a minimum of $1 \mathrm{~g}$ of hair, collected from several sites on the head, be analyzed. We still maintain, because Napoleon did not have obvious clinical signs of chronic arsenic poisoning, that the most likely source of the excess arsenic in his hair was from the use of arsenic as a preservative after collection $(7,8)$.
Nous ne croyons pas que Kintz et al (1) ont confirmé que l'arsenic trouvé dans les cheveux de Napoléon était dérivé d'une ingestion orale. Ils ont démontré que suite à un trempage de 4 heures des cheveux humains dans une solution $\mathrm{As}_{2} \mathrm{O} 3$, il est possible d'isoler l'arsenic avec une solution de lavage acétone-eau. Peut-être s'ils avaient exposé les cheveux à l'arsenic pour une période plus longue, l'arsenic n'aurait pas été enlevé. Smith et Henry (2) ont seulement été capables de récupérer $40 \%$ de l'arsenic dérivé d'un trempage de cheveux humains pendant 10 jours dans une solution d'arsenite de sodium, en lavant par la suite dans de l'eau et de l'hydroxyde de sodium. De plus, Young et Rice (3) ont été incapables de distinguer entre l'arsenic de source externe ou interne dans des poils de cochons d'Inde en lavant avec un acide, une base, de l'éthanol ou de l'éther. Atalla (4) est arrivé à la même conclusion en utilisant des cheveux humains. Nous avons démontré (5) que de l'arsenic appliqué de façon topique peut quelquefois être retrouvé au centre du cheveu humain et qu'il est difficile d'imaginer comment cette substance pourrait être accessible aux solutions de nettoyage.

Kintz et al (1) expliquent que les différences majeures dans les niveaux d'arsenic des cheveux de l'empereur analysés la même journée, certains normaux, certains létaux, sont la conséquence d'une grande variation d'arsenic inter-cheveux et intra-cheveux. Ceci est un point convaincant qui illustre la faible performance des mesures d'arsenic sur cheveu unique (6). Il est essentiel qu'un minimum de 1 gramme de cheveu soit prélevé à plusieurs endroits sur la tête et soit analysé. Nous demeurons convaincus, puisque Napoléon n'avait pas de signes cliniques évidents d'intoxication chronique à l'arsenic, que la source la plus probable de l'excès d'arsenic dans ses cheveux était l'usage d'arsenic comme agent de conservation après la collecte $(7,8)$. 


\section{Références}

1. Kintz P, Goullé JP, Fornes P, Ludes B. Une nouvelle série d'analyse des cheveux de Napoléon confirme une exposition chronique à l'arsenic. Ann de Toxicol Anal 2001 ; $13: 243-6$.

2. Smith S, Hendry EB. Arsenic in relation to the keratin tissue. Br Med J $1934 ; 2$ : 675-7.

3. Young EG, Rice FAH. On the occurrence of arsenic in human hair and its medicolegal significance. J Lab Clin Med 1944 ; 29 : 439-46.

4. Atalla LT, Silva CM, Lima FW. Activation analysis in human hair. Ann Acad Bras Cienc 1965 ; 37 : 433-41.
5. Hindmarsh JT. Caveats in hair analysis in chronic arsenic poisoning. Clin Biochem 2002 ; $35: 1-11$.

6. Cornelis R. Neutron activation analysis of hair, failure of a mission. J Radioanal Chem 1973 ; 15 : 305-16.

7. Hindmarsh JT, Corso PF. The death of Napoleon: a critical review of the cause. J Hist Med Allied Sci 1998 ; $53: 201-18$.

8. Hindmarsh JT, Corso PF. The illnesses of Napoleon Bonaparte and King George III : medical myths ? Eur J Lab Med $1999 ; 7$ : 135-43.

\section{Réponse de l'auteur}

Pascal KINTZ, IML de Strasbourg

(Reçu le 3 juin 2002 ; accepté le 7 juin 2002)

Dans le langage populaire, poison et arsenic ont été souvent synonymes.

La prudence, liée aux conclusions de l'affaire Marie Besnard, a conduit les auteurs de l'article présentant les résultats d'une nouvelle série d'analyses des cheveux attribués à l'Empereur (1) à ne jamais employer le terme d'empoisonnement, mais bien celui d'intoxication chronique.

Dans leur lettre à l'éditeur, Hindmarsh et Corso contestent à nouveau les conclusions d'investigations scientifiques.

Sur le plan clinique, peut-on conclure à une exposition chronique d'arsenic ? Certains signes rapportés par le médecin Antommarchi (2) et récemment complétés par le Dr Paul Fornes (3) sont très évocateurs, tandis que d'autres, plus classiques (accidents cutanés, bandes de Mées ...) sont manquants. C'est pourquoi, le Prof Chantal Bismuth concluait récemment (4) "le clinicien se reconnaît incapable de conclure, seuls les examens analytiques peuvent faire avancer l'énigme". Il est donc faux de dire que Napoléon n'avait pas de signes cliniques évidents d'intoxication chronique à l'arsenic. La vérité est que ces signes ne sont pas suffisants.

Plusieurs points critiques de notre expertise semblent avoir trouvé une réponse satisfaisante pour Hindmarsh et Corso, qui ne retiennent plus que le problème de la contamination externe pour expliquer la positivité des cheveux. Cela représente déjà un grand progrès par rapport à leur précédente critique (5).

Dans les expériences de contamination retenues par Hindmarsh et Corso, 3 points méritent d'être soulignés : ces références sont particulièrement anciennes et datent d'une époque où la Médecine Légale n'avait pas encore intégrée en routine les cheveux comme matrice d'investigation et donc les cinétiques de décontamination (6) ; ces études ont surtout été faites chez les rongeurs qui ne sont pas dotés de système de sudation et donc n'incorporent pas de la même façon que les humains les xénobiotiques (7) ; enfin et surtout, toutes ces études ont été faites après trempage en milieu aqueux de cheveux déjà coupés, ce qui constitue à l'évidence une voie d'introduction centrale par capillarité de l'arsenic dans la medulla (8), absolument non conforme à la réalité.

L'utilisation d'arsenic après la collecte, qui aurait pu se déposer le long de la cuticule n'est pas envisageable, car les liquides de décontamination auraient alors été fortement positifs en arsenic, ce qui n'a pas été le cas.

Nous ne contestons pas que le trempage pendant plusieurs jours des cheveux dans un bain d'arsenic puisse conduire à des résultats positifs, même après décontamination sévère. C'est d'ailleurs cette technique que nous utilisons pour préparer artificiellement des cheveux enrichis en xénobiotiques pour assurer la validation de nos méthodes (9). J'ai néanmoins de sérieux doutes sur une telle pratique pour conserver les che- 
veux de l'Empereur, d'autant que cela aurait dû être fait, dans les mêmes conditions, sur tous les échantillons disponibles (analysés depuis 1960 dans plusieurs laboratoires) et à toutes les périodes. N'oublions pas la positivité de la mèche recueillie en 1816 (1), soit 5 ans avant le décès. Enfin, cette pratique, si "courante" pour expliquer que tous les cheveux soient positifs, n'a jamais été rapportée par une quelconque personne de l'entourage de Ste Hélène.

Il est vrai qu'il existe une grande variabilité inter-cheveux, pouvant s'expliquer aisément par une phase de croissance différente, l'arsenic n'étant incorporé qu'en phase anagène (à l'opposé des phases catagène et télogène) qui représente tout de même $85 \%$ des cheveux présents sur le scalp. La multiplication des cheveux et dans notre cas des mèches, permet facilement de contourner cet aspect physiologique (10), si par malchance l'unique cheveu analysé était en phase télogène (et n'aurait donc pas incorporé d'arsenic).

Au total, que reste-t-il pour expliquer la présence d'arsenic dans les cheveux de l'Empereur? Une exposition chronique à l'arsenic par voie orale ou une contamination externe irréaliste et sans objet de tous les cheveux, à chaque coupe, pendant des années et avec le plus grand secret, dans un long bain d'arsenic.

Un mélange de doutes, de certitudes et de raisonnable ... et c'est tant mieux!

Moi, j'ai déjà fait mon choix.

\section{Références}

1. Kintz P., Goullé JP., Fornes P., Ludes B. Une nouvelle série d'analyse des cheveux de Napoléon confirme une exposition chronique à l'arsenic. Ann. Toxicol. Anal. $2001 ; 13: 243-6$.

2. Antommarchi F. Les derniers moments de Napoléon. Paris, 1825 ; vol 1.

3. Fornes P. La recherche de la cause de la mort de Napoléon 1er. In Lemaire J.F., Fornes P., Kintz P., Lentz T. eds. Autour de l'empoisonnement de Napoléon. Nouveau Monde Editions, Paris, 2001 ; 51-62.

4. Bismuth C. Napoléon est-il mort d'empoisonnement par l'arsenic ? Approche clinique. In Revue historique de la société de sauvegarde du château impérial de Pont-deBriques, ed. Etudes napoléoniennes. Levallois-Perret, $2000 ; 809-12$.
5. Corso P.F., Hindmarsh J.T., Dello Stritto F. The death of Napoleon. Am. J. Forensic Med. Pathol. 2000 ; 21 : 300-5.

6. Baumgartner W.A., Hill V.A., Blahd W.H. Hair analysis for drugs of abuse. J. Forensic Sci. 1989 ; 34 : 1433-53.

7. Cone E.J. Mechanisms of drug incorporation into hair. Ther. Drug Monit. 1996 ; 18 : 438-43.

8. Pötsch L. A discourse on human hair fibers and reflections on the conservation of drug molecules. Int. J. Leg. Med. 1996 ; 108 : 285-93.

9. Cirimele V., Kintz P., Staub C., Mangin P. Testing human hair for flunitrazepam and 7-aminoflunitrazepam by GC/MS/NCI. Forensic Sci. Int. 1997 ; 84 : 189-200.

10. Kintz P. Matrices alternatives et toxicologie. In Kintz P. coordinateur. Toxicologie et pharmacologie médicolégales. Elsevier, Paris, 1998 ; 685-710. 